Voix et Images

\title{
De la nostalgie d'un monde possible à la possible fin du monde
}

\section{Pascal Riendeau}

Volume 35, numéro 1 (103), automne 2009

Herménégilde Chiasson

URI : https://id.erudit.org/iderudit/038575ar

DOI : https://doi.org/10.7202/038575ar

Aller au sommaire du numéro

\section{Éditeur(s)}

Université du Québec à Montréal

\section{ISSN}

0318-9201 (imprimé)

1705-933X (numérique)

Découvrir la revue

Citer ce compte rendu

Riendeau, P. (2009). Compte rendu de [De la nostalgie d'un monde possible à la possible fin du monde]. Voix et Images, 35(1), 120-125.

https://doi.org/10.7202/038575ar d'utilisation que vous pouvez consulter en ligne.

https://apropos.erudit.org/fr/usagers/politique-dutilisation/ 


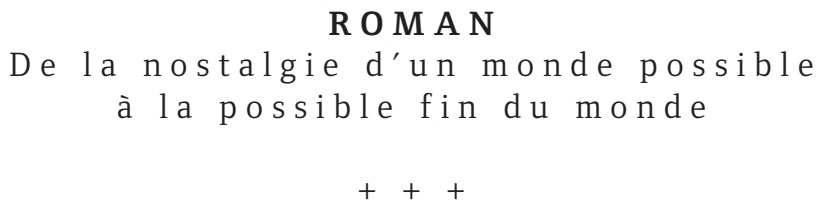

PASCAL RIENDEAU

Université de Toronto

- Jack a presque fini son livre. Il a trouvé un titre: L'anglais n'est pas une langue magique. C'est une phrase que j'avais prononcée devant lui.

- Drôle de titre pour un roman ${ }^{1} \ldots$

Celui qui a soufflé le titre à Jack Waterman, c'est son petit frère Francis, personnage jusqu'alors inconnu de l'univers romanesque de Jacques Poulin et narrateur de L'anglais n'est pas une langue magique; celle qui s'étonne, c'est Marine, la traductrice attitrée de l'œuvre de Jack et narratrice du roman précédent de l'auteur, La traduction est une histoire d'amour. Peu de temps après cet échange, le vieux Jack se montre pourtant «insatisfait du roman qu'il venait de terminer [-] il en avait commencé un autre dont il ne voulait rien dire pour l'instant» (155). Le recours à la mise en abyme, même de façon lacunaire, est un procédé récurrent chez Poulin, mais alors ici, quel roman lit-on? Est-ce celui de Jack ou le roman insatisfaisant récupéré par le petit frère? A-t-on entre les mains une œuvre périphérique, en attendant le véritable grand roman de Jack? D'ailleurs, à quoi tenait son insatisfaction? En partie au fait que pour écrire son grand roman de l'Amérique française, il a dû recourir à un livre traduit de l'anglais: La piste de l'Ouest. Journal de la première traversée du continent nord-américain de Meriwether Lewis et William Clark. Ah, le grand roman de l'Amérique française! Il s'agit d'une constante dans l'œuvre de Poulin; elle remonte au moins au roman Les grandes marées (1978), qui met en scène un traducteur vivant seul sur une île progressivement envahie par des personnages censés venir l'aider à sortir de son isolement. Un de ceux-là est l'Auteur, un personnage maussade et incapable d'écrire ; il caressait un projet littéraire qu'il résumait ainsi :

le roman français s'intéresse plutôt aux idées, tandis que le roman américain s'intéresse davantage à l'action. Or, nous sommes des Français d'Amérique, ou des Américains d'origine française, si vous aimez mieux. Nous avons donc la possibilité,

$$
+++
$$

1 Jacques Poulin, L'anglais n'est pas une langue magique, Montréal/Arles, Leméac/Actes Sud, 2009, p. 145. 
au Québec, d'écrire un roman qui sera le produit de la tendance française et de la tendance américaine. C'est ça que j'appelle le grand roman de l'Amérique².

Il ne faudrait pas minimiser l'importance de l'ironie entourant cette proposition qui rassemble beaucoup de lieux communs. On ne peut toutefois pas l'exclure du projet romanesque de Poulin, même si elle se présente de façon plus nuancée, lorsqu'il arrive à Poulin d'en parler. Après douze romans, on constate qu'il a montré à la fois l'impossibilité d'écrire ce grand roman de l'Amérique (française) tel que l'Auteur des Grandes marées le décrit, tout en continuant à annoncer son intention d'y parvenir. Dans un (rare) entretien avec Christian Desmeules, Poulin a reconnu avoir «comme une sorte de nostalgie de ce que ç'aurait pu être ${ }^{3}$ ", cette grande présence française en Amérique. Dans L'anglais n'est pas une langue magique, Jack Waterman admet à son frère que son désir d'écrire ce grand récit épique ne pourra jamais se réaliser. Comment composer "une sorte d'épopée» quand les mots "venaient au comptegouttes» (110)? Il n'en reste pas moins que Poulin en a proposé de multiples versions, plus modestes, mais certainement originales, que ce soit Les grandes marées, Volkswagen blues ou maintenant L'anglais n'est pas une langue magique. Ce dernier montre en quelque sorte l'épuisement de cette idée qui entraîne l'incomplétude du roman de Jack, même si elle semble rester présente sous la forme d'un fantasme permanent.

Jack Waterman est bien entendu le personnage d'écrivain qu'on retrouvait déjà dans plusieurs romans antérieurs - un vague alter ego de l'auteur -, et son refus de nous laisser entrer dans son nouvel opus s'avère une façon de nous détourner de ce qui aurait pu devenir un roman d'écrivain, à l'instar du Vieux chagrin. L'apparente naïveté de l'énoncé du titre - L'anglais n'est pas une langue magique - n'est pas fortuite, car elle émane d'un narrateur candide dont le ton bon enfant marque l'ensemble du texte. Après le roman de la traductrice (La traduction est une histoire d'amour), qui faisait de l'écrivain un personnage secondaire, Poulin propose le roman du lecteur, qui laisse Jack encore plus dans l'ombre. Il s'agit donc d'un second roman décentré, c'est-à-dire qui confine l'écrivain à un rôle mineur dans l'action du récit, mais dont la présence implicite reste essentielle. En réalité, ce décentrement fait ressortir davantage l'importance de Jack Waterman dans l'œuvre de Poulin. Cependant, on peut croire que ses lecteurs fidèles souhaiteront sans doute retrouver le vieux Jack, devenu plus cynique et plus misanthrope, qui se décrit comme une "ruine ambulante» et un "vieux chialeux». Francis, jeune frère gentil, généreux et dévoué, ne veut pas faire de peine à ceux qu'il aime; ses propos, certes non exempts de mièvrerie, placent le roman dans un équilibre précaire entre une vision du monde singulière et une histoire pleine de beaux et bons sentiments.

L'anglais n'est pas une langue magique devient l'occasion pour Poulin de reconstruire a posteriori la famille de Jack Waterman, qui a maintenant 50 ans - il

$$
+++
$$

2 Jacques Poulin, Les grandes marées, Montréal/Arles, Leméac/Actes Sud, coll. «Babel», 1995 (1978), p. 177.

3 Christian Desmeules, "Jacques Poulin, le roman et l'art du rapaillage», Le Devoir, samedi 21 et dimanche 22 mars 2009, p. F3. 
se décrit pourtant comme un vieillard sentant la mort s'approcher -, alors qu'il avait déjà 40 ans dans Volkswagen blues, paru en 1984. Il ne faut sans doute pas chercher une adéquation parfaite entre les dates, les événements et les personnages de l'ensemble de l'univers de Poulin. Dans son dernier roman, il procède à un bilan de son œuvre - quitte à créer une série de problèmes chronologiques -, en ajoutant un autre point de vue, un nouveau narrateur. En général, le protagoniste chez Poulin, qu'il soit écrivain ou traducteur, lecteur ou bibliophile, se définit presque toujours par trois types de relations: avec la lecture ou l'écriture, avec une femme qu'il peut difficilement séduire et avec un frère cadet. La relation fraternelle est cette fois-ci renversée, car c'est le jeune frère qui occupe l'avant-scène. Francis ressemble néanmoins aux protagonistes habituels de Poulin, mais en plus doux, plus naï, plus simple. Il aime les mêmes auteurs que son frère (Hemingway, Gabrielle Roy), et la générosité et la compassion l'habitent. Lecteur professionnel, il offre ses services à ceux qui ne peuvent pas ou plus lire, faisant de la lecture une activité thérapeutique originale dont il doute de l'efficacité réelle, et ce, même si une jeune fille dans le coma à qui il lisait régulièrement L'avalée des avalés s'est réveillée brièvement, laissant croire que sa thérapie ne se révélait peut-être pas si vaine.

Son succès le plus évident demeure toutefois le rétablissement d'une de ses auditrices, Limoilou, la jeune fille que Marine a hébergée dans La traduction est une histoire d'amour. La force thérapeutique des lectures de Francis aurait grandement aidé Limoilou à reprendre goût à la vie après une tentative de suicide. Elle quitte le chalet de Marine sur l'île d'Orléans après environ un an pour aller habiter seule à Québec. Afin de célébrer sa nouvelle liberté, elle procède à une étonnante cérémonie qui consiste à laisser bien en vue dans des lieux publics un exemplaire des livres qui l'ont marquée: L'appel de la forêt de Jack London, La route d'Altamont, Salut Galarneau! et Lumière des oiseaux de Pierre Morency. Pourquoi ces quatre livres-là? Risquons une hypothèse: s'ils ne forment pas un ensemble homogène, on peut y voir une véritable cohérence et les imaginer comme un quatuor qui donne une certaine couleur à la façon d'explorer, de nommer, d'écrire et de décrire l'espace nordaméricain. Le roman de Poulin est un hommage à la littérature ou plus particulièrement à la lecture, qui se manifeste également d'une autre façon: L'anglais n'est pas une langue magique se présente comme une lecture de deux ouvrages, La piste de l'Ouest. Journal de la première traversée du continent nord-américain, cité de nombreuses fois par Poulin, mais aussi America de Denis Vaugeois - lui-même une lecture du journal de Lewis et Clark - , intégré plus discrètement au récit. Ainsi, Poulin réussit à inventer en quelque sorte le roman d'une improbable rencontre entre une épopée et un récit intimiste.

Le dernier roman de Nicolas Dickner, Tarmac ${ }^{4}$, recrée un univers qui n'est pas sans rappeler celui du précédent, Nikolski; le récit commence aussi en 1989, dure une

$$
+++
$$

4 Nicolas Dickner, Tarmac, Québec, Alto, 2009, 274 p. 
douzaine d'années et présente des personnages à la fin de l'adolescence aux prises avec des fixations étranges - cette fois-ci, ce n'est pas pour les déchets, mais pour une apocalypse imminente. Dans son second roman, Dickner donne l'impression d'avoir repris une des leçons de Poulin tirées des commentaires sur l'écriture d'Hemingway: aller vers la simplicité. En effet, les phrases tendent au dépouillement, les dialogues sont plus nombreux, l'intrigue, moins complexe, le récit, plus linéaire et les chapitres, courts; ceux-ci sont tous coiffés - comme chez Poulin d'un titre descriptif, parfois humoristique. Cela dit, l'attrait pour l'étrange ou l'invraisemblable reste analogue à celui de Nikolski, et la science est l'un des principaux discours qui façonnent la texture romanesque. Ce qui distingue plus particulièrement Tarmac, c'est cette fascination pour la fin du monde; le roman met en place ce que Jean-François Chassay appelle un «imaginaire de la fin [qui] concerne des représentations d'un monde sur le point de finir ${ }^{5}$ ".

Dickner ne conçoit pas un univers très familier qu'il s'ingénie par la suite à bouleverser par la venue d'un personnage marginal. Dès l'ouverture de Tarmac, le loufoque et l'ordinaire se côtoient. L'arrivée par hasard de Hope Randall et de sa mère à Rivière-du-Loup à la fin de l'été 1989 constitue l'élément déclencheur du récit que nous livre le narrateur, Michel Bauermann, adolescent de 17 ans qui ressent immédiatement une admiration pour cette inconnue qui l'aborde en lui racontant le récit du rêve qu'elle a fait de la bombe d'Hiroshima, accompagné d'une dissertation sur la bombe, rigoureusement scientifique dans la méthode, mais dont l'illustration étonne. Une relation d'amitié basée sur des affinités électives se développe rapidement entre les deux, et ils resteront inséparables jusqu'à ce que Hope parte à la recherche d'un mystérieux prophète qui a prédit - tout comme elle - la fin du monde pour le 17 juillet 2001. Tarmac contient donc trois récits: le premier raconte la relation d'amitié entre Michel et Hope, de l'été 1989 au printemps 1991; le deuxième se concentre sur le périple de Hope à travers l'Amérique du Nord, puis sur son installation au Japon; le troisième se déroule douze ans après leur première rencontre et décrit les moments qui précèdent et qui suivent la date fatidique, durant lesquels un Michel Bauermann presque trentenaire et nostalgique cherche désespérément des nouvelles de son ancienne amie.

L'originalité de la mise en place d'un «imaginaire de la fin» vient de cette conjonction entre le discours social d'une époque (la peur d'une apocalypse nucléaire durant la fin de la guerre froide) et un groupe d'illuminés qui prédit la fin du monde depuis au moins 1835. Il s'agit non pas d'une secte religieuse, mais d'une simple famille d'Acadiens (les Randin devenus Randall) déportés en 1755 puis revenus en Nouvelle-Écosse, jamais assimilés et se mêlant peu au reste de la population. Contrairement à ses ancêtres qui reçoivent la révélation de la date de la fin du monde durant un rêve, Hope la découvre grâce à un coup de dés; voulant prouver qu'Einstein avait tort d'affirmer: «Dieu ne joue pas aux dés», Hope croit au contraire qu'elle peut déterminer la date de la fin du monde à l'aide du hasard. Cette date

$$
++
$$

5 Jean-François Chassay, Dérives de la fin. Sciences, corps \& villes, Montréal, Le Quartanier, coll. «Erres Essais», 2008, p. 9. 
fatidique devient rapidement une veritable obsession; c'est ce qui incite Hope à quitter Rivière-du-Loup pour se rendre à New York, puis parcourir les États-Unis d'est en ouest jusqu'à Seattle à la recherche du fameux prophète Charles Smith. On constate à nouveau chez Dickner un intérêt pour la traversée du continent nordaméricain, mais celle de Tarmac se veut elliptique et ironique, et contrairement à ce que fait Poulin dans Volkswagen blues, par exemple, elle n'est pas accompagnée de considérations sur l'histoire ou la géographie. La description détaillée du territoire est remplacée par quelques brèves répétitions qui montrent la banalité et l'uniformité : «Champ de maïs, cours à métaux, champ de soya, champ de maïs, incinérateur, cinéparc, parc industriel, Wal-Mart, champ de maïs, concessionnaire Ford, champ de maïs, motel [...].» (158) Chez Dickner, l'ironie touche moins directement les personnages que leur environnement ou les objets qui les entourent et les définissent. La conscience de Hope ou son état d'esprit durant ce long voyage sont remplacés par une liste d'une page des aliments qui composent sa nourriture quotidienne, gracieuseté des machines distributrices. Le «Menu du voyageur» - titre du 55e chapitre est constitué de "stéaroyl-2-lactylate de sodium, farine de maïs, phosphate de calcium [...], gluten de blé, alcool éthylique, sorbitol» (159) et de toute une variété d'ingrédients qui nous donnent presque l'impression de lire une variante de l'exploréen. Arrivée au Japon, elle rencontre, après d'innombrables tentatives, le prophète Charles Smith (qui est en fait le pseudonyme du Japonais Hayao Kamajii), qui disparaît mystérieusement après avoir expliqué en détail - et en français - dans quelles circonstances lui était apparue sa vision de la fin du monde. Comment le narrateur sait-il ce qui se passe au Japon puisque Hope ne le contacte plus après son départ des États-Unis? Le récit qu'il fait de sa vie au Japon est peu vraisemblable du point de vue de la logique narrative, mais l'invraisemblable fait aussi partie intégrante du récit; d'ailleurs, depuis qu'elle était au Japon, Hope «se découvrait [...] une capacité croissante à tolérer l'invraisemblable et l'improbable» (186).

La préoccupation de Michel pour la fin du monde s'avère beaucoup plus modeste si on la compare à l'obsession de Hope. Dans l'ensemble du roman, il conserve une certaine distance ironique, il se fait davantage le témoin de celle qui est habitée par cette idée fixe. Les discours autour de l'apocalypse qui foisonnent deviennent une façon de mettre en jeu un savoir hybride, hétéroclite; on pourrait le nommer le savoir de l'inessentiel. Par exemple, après avoir fait l'expérience de «bricoler une pile électrique avec un citron», Hope procède à des calculs mathématiques afin d'apprendre combien de mégacitrons seraient nécessaires pour recréer une bombe atomique. Commentant cet épisode loufoque, Dickner a déclaré à Caroline Montpetit: "Il ne faut pas se fier à la science pour expliquer le monde ${ }^{6}$ ", laissant probablement sous-entendre que ce rôle revenait à la littérature. Il n'empêche que les explications scientifiques (vérifiables ou non) reprises dans Tarmac enrichissent le roman, surtout quand leur intégration survient à des moments inattendus, comme lorsque Michel regarde Hope dormir: «Le cerveau humain consom-

6 Caroline Montpetit, «Nicolas Dickner: l'Apocalypse n'est pas pour demain», Le Devoir, 18 et 19 avril 2009 , p. F1. 
mait prétendument le cinquième de l'énergie produite par le corps, mais celui de Hope en flambait visiblement davantage - et tandis qu'elle respirait tout doucement, les yeux clos, j'imaginais son cortex fissionner en silence des noisettes d'uranium 235.» (48) En plus de recourir à la science, le narrateur multiplie les commentaires sur le monde qui l'entoure, en tire quelquefois des maximes. Vers la fin du roman, après une ellipse narrative de dix ans, il entame une réflexion sur son passé: «J'ai grandi dans un monde obsédé par l'apocalypse. [...] Nous voyions la fin du monde partout. Même un banal changement de date nous paraissait susceptible de provoquer l'effondrement de la civilisation [...].» (244-245) Et le tarmac, dans tout cela? De la plateforme aéroportuaire de Mirabel, Michel Bauermann le regarde avant de s'envoler pour Tokyo y rejoindre Hope; le tarmac devient alors un lieu d'observation, un reflet du monde entre le passé et l'avenir, entre la fin d'un monde et le monde nouveau: «Moi, j'étais plutôt content de m'envoler par Mirabel. Avec les rumeurs croissantes de fermeture, je me sentais en visite parmi des ruines virtuelles - le dosage idéal d'archéologie et de science-fiction.» (267)

D'autres caractéristiques de l'univers romanesque de Dickner rappellent celui de Poulin; on peut penser à la conception de l'amitié entre un homme et une femme, au traitement de la sexualité, ainsi qu'à une certaine pudeur. Mais là s'arrêtent sans doute les comparaisons. Avec L'anglais n'est pas une langue magique, Poulin montre qu'il se rapproche du bilan d'une grande œuvre qui se tourne de plus en plus vers elle-même. Le grand roman de l'Amérique auquel rêve Poulin prend un sens tout à fait différent chez Dickner, qui est également, à sa façon, un explorateur du territoire nord-américain. Bien que la forme qu'il propose dans Tarmac ne soit pas la plus audacieuse, il réussit à illustrer les contradictions d'une époque obsédée par les discours de la fin, faisant aussi de la science une matière particulièrement riche pour le roman. 